\title{
Renal cell carcinoma and amyopathic dermatomyositis
}

\section{Anthony F. Adili, MBBCh(C); Haralambos Liaconis, MD, FRCSC; Kaela Gusenbauer; Anil Kapoor, MD, FRCSC}

Section of Urology, Department of Surgery, McMaster University, Hamilton, ON

Cite as: Can Urol Assoc J 2015;9(5-6):E340-2. http://dx.doi.org/10.5489/cuaj.2362 Published online May 13, 2015.

\section{Abstract}

Amyopathic dermatomyositis (dermatomyositis siné myositis) is a rare disorder with cutaneous lesions identical to those of classic dermatomyositis, but with no clinical evidence of myopathy. Association with malignancy has been suggested in a number of reports. We report the case of a 69-year-old man with a recent diagnosis of amyopathic dermatomyositis, who presented to the urology service with hematuria. Investigations revealed a 5.8$\mathrm{cm}$ renal cell carcinoma and the patient underwent left radical nephrectomy without complication. To our knowledge, an association with renal cell carcinoma and amyopathic dermatomyositis has not been previously described.

\section{Introduction}

Dermatomyositis (DM) is described as both a progressive primary inflammatory myopathy and an idiopathic microangiopathic disorder. ${ }^{1,2}$ DM is believed to be marked by immune-mediated chronic inflammation resulting in symmetrical proximal muscle weakness and a distinctive skin rash. ${ }^{3,4}$ The primary skin change is a confluent or patchy macular violaceous erythema, as well as pruritus. ${ }^{3}$ Characteristic skin manifestations include heliotrope rash, periungual telangiectasia, edema, and facial erythema, with the hallmark being Gottron's papules - scaling, erythematous eruptions over the knuckles and other extensor surfaces. ${ }^{3-5}$ Amyopathic dermatomyositis (ADM), first coined by Pearson in 1963, is a rare but well-recognized clinical subtype of DM, constituting $2 \%$ to $18 \%$ of patients with this disease. ${ }^{6}$ Diagnostic criteria for ADM are cutaneous lesions and skin biopsy results indistinguishable from DM, but with no clinical evidence of proximal muscle weakness or serum muscle enzyme abnormalities for at least 6 months..$^{2-4,6,7}$
The association between internal malignancy and DM has been reported, with reports as high as $60 \%{ }^{6}$ Carcinomas can affect almost any organ system, however in DM they predominantly involve the reproductive organs, pulmonary system, and gastrointestinal tract. ${ }^{3,4,8,9} \mathrm{~A}$ similar association has been demonstrated with ADM although it remains controversial with interstitial lung disease being the mainstay. ${ }^{2-4,8,9}$ A detailed literature search revealed no cases of renal cell carcinoma associated with ADM. We report a case of renal cell carcinoma associated with ADM in a 69-yearold man who presented with hematuria.

\section{Case report}

A 69-year-old man presented to the dermatology service with erythematous papules on the dorsum of both hands and a rash over the face and eyelids. Although less symptomatic, the patient described similar lesions occurring frequently over the past 15 years. His medical history was significant for hypertension, osteoporosis, and symptoms of gastroesophageal reflux disease without dysphagia. Musculoskeletal examination was completely normal. Punch biopsy of the hand lesions revealed cellular changes consistent with dermatomyositis (DM). The patient was referred to a rheumatologist for further investigation. Routine laboratory tests, including CPK, ANA, anti-ENA, protein electrophoresis, and urinalysis, were normal. Pulmonary function tests were unremarkable and an electromyographic study was negative for myopathy.

A few weeks later his rash worsened and Gottron's nodules were identified on the metacarpophalangeal and proximal interphalangeal joints of both hands. The patient was treated with a 3-month tapering course of the corticosteroid prednisone and the antimalarial agent hydroxychloroquine. His condition improved, and after 9 months of investigations and treatment with no clinical or laboratory evidence of muscle involvement, a clinical diagnosis of ADM was made.

Two months later, the patient presented to the urology service with a 2-week history of painless, gross hematuria 
with spontaneous resolution. The patient was a non-smoker with no history of renal disease. Laboratory investigations were all within normal range, except for mild microcytosis. Urine microscopy revealed 5-10 RBC/hpf. Ultrasound of the abdomen and pelvis revealed a $5.8-\mathrm{cm}$ diameter isoechoic mass in the upper pole of the left kidney. The patient was referred for computed tomography (CT) of the abdomen and pelvis as well as a bone scan, chest X-ray, and serum liver function tests. The CT scan showed a $4.7 \times 5.8-\mathrm{cm}$ mass arising from the upper pole of the left kidney with no synchronous lesion or evidence of spread outside Gerota's fascia; however, the tumour extended along the left renal vein. All other investigations were normal.

With no contraindications to surgery, the patient underwent an uncomplicated left radical nephrectomy. The renal vein thrombus was removed entirely with the specimen and there was no tumour spillage or violation of Gerota's fascia. Pathologic examination showed a clear cell carcinoma (Fuhrman grade 3/4) with all margins negative for malignancy. The adrenal gland and peri-aortic lymph nodes taken for sampling were also negative for malignancy.

The patient did well postoperatively and was followed in the outpatient clinics by both the urology and rheumatology departments. The patient is currently feeling well with no evidence of malignancy, myopathy, and resolution of his cutaneous ADM lesions.

\section{Discussion}

$\mathrm{ADM}$ is a rare disease, with an incidence of only $2 \%$ to $18 \%$ that of classic DM (higher in the Asian population). ${ }^{3,6}$ It has a

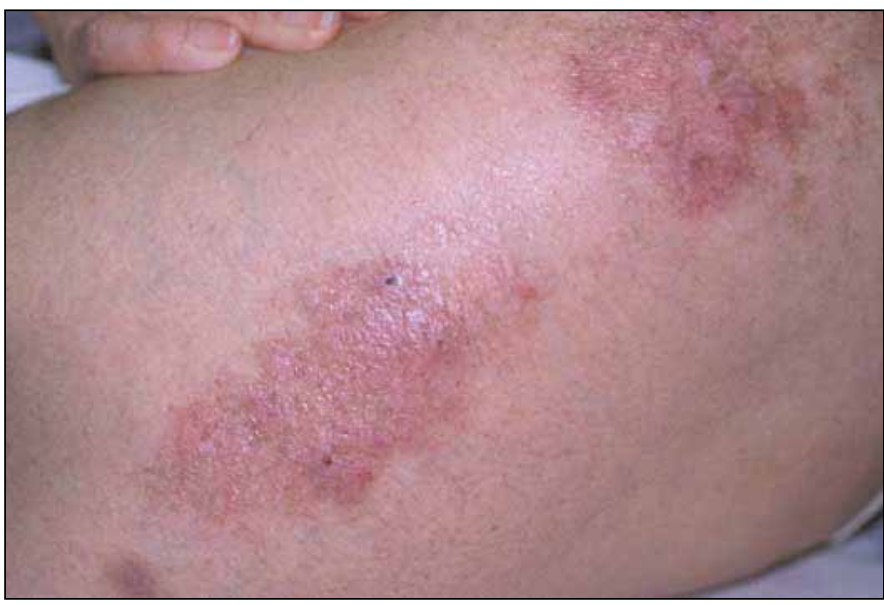

Fig. 1. Cutaneous dermatomyositis is depicted as a patchy, macular, violaceous erythema classically displaying a livedo or reticulated array. This cutaneous change predominantly occurs over the upper thighs, and lateral aspect of the hips and has such been designated as the holster sign. (From "Sontheimer $\mathrm{RD}$. Dermatomyositis: an overview of recent progress with emphasis on dermatologic aspects. Dermatol Clin 2002;20:387-408. Reprinted with permission.)
3:1 female preponderance, and most cases do not progress to myopathy or a diagnosis of DM..$^{4,6}$ An association with malignancy similar to that of classic DM has been described in the literature. . $^{3,4,6,8,9}$

The exact relationship between malignancy and ADM and DM has yet to be demonstrated. Confounding factors include the lack of a formal classification for ADM, variations in the timing of ADM diagnosis relative to the detection of cancer, and the effects of medical therapy. ${ }^{2-4}$

One theory is that DM and ADM represent paraneoplastic syndromes, where factors from a pre-existing tumour trigger an inflammatory or immune-mediated response leading to cutaneous manifestations, with or without the myopathy. ${ }^{7,9}$ However, this theory is controversial for several reasons, including the fact that a diagnosis of ADM often predates the detection of any malignancy.

In this case, the patient exhibited absolutely no signs or symptoms of renal cell carcinoma when he presented to a dermatologist with a symptomatic rash. Initially, a working diagnosis of DM was made based on hallmark cutaneous lesions and biopsies consistent with classic DM. However, the final diagnosis was changed to ADM 9 months later after all investigations failed to reveal any evidence of muscle involvement. A focused evaluation for malignancy was not performed. We hypothesize that an undetected renal cell carcinoma may have been present for some time prior to the development of symptomatic skin lesions. The progression of this tumour, by virtue of its natural history or perhaps the medical treatment of the cutaneous lesions, may have led to the development of ADM as a paraneoplastic phenomenon.

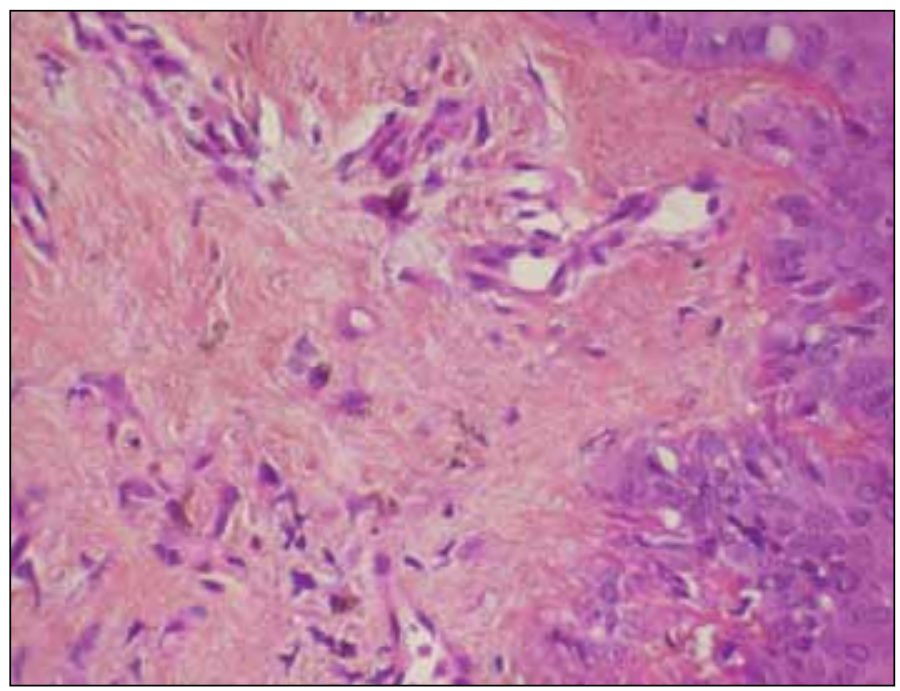

Fig. 2. Section of a skin biopsy consistent with the histologic signs of dermatomyositis. Edema, telangiectases, a moderate polymorphic inflammatory infiltrate, and melanin clumps are noted in the dermis. (From "Saoud B, Allali F, Hassouni NH. Amyopathic Dermatomyositis. Joint Bone Spine 2006;73(3):318-20. Reprinted with permission.) 
Adili et al.

\section{Conclusion}

Several studies have associated internal malignancies with both DM and ADM. Though the exact relationship is unknown, these entities may represent paraneoplastic syndromes. We recommend a high index of suspicion for malignancy, with consideration specifically to renal cell carcinoma, in patients who present with ADM.

Competing interests: Dr. Adili, Dr. Liaconis and Dr. Gusenbauer all declare no competing financial or personal interests. Dr. Kapoor is a member of the Speakers bureau for, and has received grants and honoraria from, Pfizer Oncology, GSK Oncology, Novartis Oncology and Amgen. He has also participated in clinical trials within the past 2 years with NCIC, Pfizer, GSK, Novartis and Amgen.

This paper has been peer-reviewed.

\section{References}

1. Vinay K, Cotran RS, Robbins SL. Disorders of skeletal muscle. In: Vinay K, Abbas AK, Aster JC, editors. Robbins Basic Pathology. 9th ed. Philadelphia: Saunders; 2013:805.
2. Khambatta S, Wittich CM. Amyopathic dermatomyositis. Mayo Clin Proc 2010;85:e82. http://dx.doi. org/10.4065/mcp.2010.0514

3. Sontheimer RD. Dermatomyositis: An overview of recent progress with emphasis on dermatologic aspects. Dermatol Clin 2002;20:387-408. http://dx.doi.org/10.1016/S0733-8635(02)00021-9

4. Euwer RL, Sontheimer RD. Amyopathic dermatomyositis: A review. I Invest Dermatol 1993;100:1245-75. http://dx.doi.org/10.1038/iid.1993.35

5. Sontheimer RD. The management of dermatomyositis: Current treatment options. Expert Opin Pharmacother 2004;5:1083-99. htrp://dx.doi.org/10.1517/14656566.5.5.1083

6. Arnson $Y$, Dovrish $Z$, Hadari R, et al. Amyopathic dermatomyositis - An uncommon presentation of dermatomyositis. Isr Med Assoc J 2007;9:492-3.

7. Maoz $C R$, Langevitz $P$, Livneh $A$, et al. High incidence of malignancies in patients with dermatomyositis and polymyositis: An 11-year analysis. Semin Arthritis Rheum 1998;27:319-24. http://dx.doi. org/10.1016/S0049-0172(98)80052-8

8. El-Azhary RA, Pakzad SY. Amyopathic dermatomyositis: Retrospective review of 37 cases. J Am Acad Dermatol 2002;46:560-5. http://dx.doi.org/10.1067/mid.2002.120620

9. Whitmore SE, Watson R, Rosenshein NB, et al. Dermatomyositis sine myositis: Association with malignancy. J Rheumatol 1996;23:101-5

10. Saoud B, Allali F, Hassouni NH. Amyopathic dermatomyositis. Joint Bone Spine 2006;73:318-20. http:// dx.doi.org/10.1016/i.jbspin.2005.05.004

Correspondence: Dr. Anil Kapoor, Associate Professor, McMaster University, Urology, Chair, GenitoUrinary Oncology Program, Juravinski Cancer Centre, St. Joseph's Healthcare Hamilton, McMaster Institute of Urology, 50 Charlton Ave. E., Room G344, Hamilton, ON L8N 4A6; akapoor@mamaster.ca 